\title{
Electron Trapping and Detrapping in an Oxide Two-Dimensional Electron Gas: The Role of Ferroelastic Twin Walls
}

\author{
Shashank Kumar Ojha ${ }^{*}$, Sankalpa Hazra®, Prithwijit Mandal@, Ranjan Kumar Patel®, \\ Shivam Nigam, Siddharth Kumar®e, and S. Middey® ${ }^{\dagger}$ \\ Department of Physics, Indian Institute of Science, Bengaluru 560012, India
}

(Received 24 December 2020; revised 6 April 2020; accepted 13 April 2021; published 5 May 2021)

\begin{abstract}
The choice of electrostatic gating over the conventional chemical doping for phase engineering of quantum materials is attributed to the fact that the former can reversibly tune the carrier density without affecting the system's level of disorder. However, this proposition seems to break down in field-effect transistors involving $\mathrm{SrTiO}_{3}$ (STO)-based two-dimensional electron gases. Such peculiar behavior is associated with electron trapping under an external electric field. However, the microscopic nature of the trapping centers remains an open question. In this paper, we investigate electric-field-induced chargetrapping and charge-detrapping phenomena at the conducting interface between the band insulators $\gamma-\mathrm{Al}_{2} \mathrm{O}_{3}$ and STO. Our transport measurements reveal that the charge trapping under a positive back-gate voltage $\left(V_{g}\right)$ above the tetragonal-to-cubic structural transition temperature $\left(T_{c}\right)$ of STO has a contribution from electric-field-assisted thermal escape of electrons from the quantum well, and from clustering of oxygen vacancies as well. We observe an additional source of trapping below $T_{c}$, which arises from the trapping of free carriers at ferroelastic twin walls in the STO. Application of a negative $V_{g}$ results in charge detrapping, which vanishes above $T_{c}$. This feature demonstrates the crucial role of structural domain walls in the electrical transport properties of STO-based heterostructures. The number of charges trapped (detrapped) at (from) a twin wall is controlled by the net polarity of the wall and is completely reversible with a sweep of $V_{g}$.
\end{abstract}

DOI: 10.1103/PhysRevApplied.15.054008

\section{INTRODUCTION}

The field-effect transistor (FET), based on the principle of electrostatic gating of semiconductor-based heterostructures, is the foundation of modern electronics. In order to achieve higher transistor packing densities beyond the scaling limitations of silicon-based FETs, several alternative classes of materials are being explored [1-3]. A quasitwo-dimensional electron gas (2DEG) confined at the surface of a complex oxide or at an interface between complex oxides is one such promising platform [4,5]. Such oxidebased 2DEG systems host several unique electronic and magnetic phenomena such as ferromagnetism, multiband superconductivity, spin-orbital texture, and the topological Hall effect [6-13], which can further be tuned by the application of an electric field [14-17]. Owing to the very large dielectric constant of $\mathrm{SrTiO}_{3}$ (STO) [18-20], electric field control of STO-based 2DEG heterostructures can be achieved through a back-gating configuration (see Fig. 1) [14,15,21-24]. In this geometry, the conducting interface itself acts as one plate of a capacitor, and an electrode attached to the bottom of the substrate acts as the

*shashank@iisc.ac.in
${ }^{\dagger}$ smiddey@iisc.ac.in other plate. Thus, it is expected that the carrier density in the 2DEG channel can be reversibly tuned by varying the back-gate voltage $\left(V_{g}\right)$, as the application of a positive (negative) $V_{g}$ dopes electrons (holes) into the $2 \mathrm{DEG}$ channel. Surprisingly, an irreversibility in the sheet resistance $\left(R_{S}\right)$ was observed in the case of several STO-based 2DEG systems when $V_{g}$ was repeatedly swept between zero and a positive value [22-25]. Such an irreversibility in $R_{S}$ appears due to the loss of conduction electrons from the 2DEG under a positive $V_{g}$, leading to a gradual increase in $R_{s}$ with time under a fixed positive $V_{g}$. However, there is no consensus about the microscopic origin of this peculiar behavior, and mechanisms such as the thermal escape of electrons from a quantum well under an electric field [22] and the trapping of carriers in the midgap states formed due to electric-field-induced clustering of oxygen vacancies (OVs) [24] have been proposed.

While the number of trapped charges in the abovementioned processes depends on the level of structural defects present in the STO substrate and of defects (such as OVs) created at the film/substrate interface during growth $[26,27]$, there can be another inherent source of charge trapping in STO. It is well established that STO undergoes a cubic-to-tetragonal ferroelastic phase transition around $T_{c}=105 \mathrm{~K}$ [28], leading to a dense network of twin walls 
along certain crystallographic directions. The presence of an intrinsic strain gradient along with rotopolar and trilinear coupling in the tetragonal phase makes these twin walls intrinsically polar [29-32]. These polar twin walls can trap a significant amount of conduction electrons from the 2DEG $[33,34]$. Owing to its intrinsic polarity, an electric field could be an effective tool to tune further the static polarization of such twin walls, embedded in a nonpolar matrix of STO [35], and could have a dramatic impact on the macroscopic electrical-transport behavior. However, a signature of such nanoscale charge-trapping centers has not been demonstrated so far in electrostatic-gating experiments.

In this paper, we focus on electric-field-induced chargetrapping phenomena in a $\gamma-\mathrm{Al}_{2} \mathrm{O}_{3} / \mathrm{SrTiO}_{3}(\mathrm{GAO} / \mathrm{STO})$ heterostructure around the ferroelastic transition of STO. GAO has a cubic spinel structure with a lattice constant of $7.911 \AA$, almost twice the lattice constant of STO $(3.905 \AA)$. The excellent match between the oxygen sublattices of these compounds along [001] facilitates high-quality epitaxial growth of GAO on an STO substrate, and a GAO/STO heterostructure exhibits the highest mobility among all STO-based 2DEGs realized without modulation doping [36]. In contrast to the polar catastrophe-driven 2DEG in the $\mathrm{LaAlO}_{3} / \mathrm{STO}$ (LAO/STO) interface, the conducting interface in GAO/STO emerges due to the creation of OVs on the STO side. The presence of excess OVs in GAO/STO $[37,38]$ makes it more susceptible to charge trapping in back-gating experiments and needs special attention. We find that the charge trapping in this heterostructure cannot be accounted for solely by the existing proposed mechanisms for LAO/STO heterostructures. Apart from the loss of carriers due to both thermal escape and OV clustering under the application of a positive $V_{g}$, there is an additional component. Most importantly, this contribution vanishes above $100 \mathrm{~K}$ and increases with an increase in the applied field. Application of a negative $V_{g}$ results in
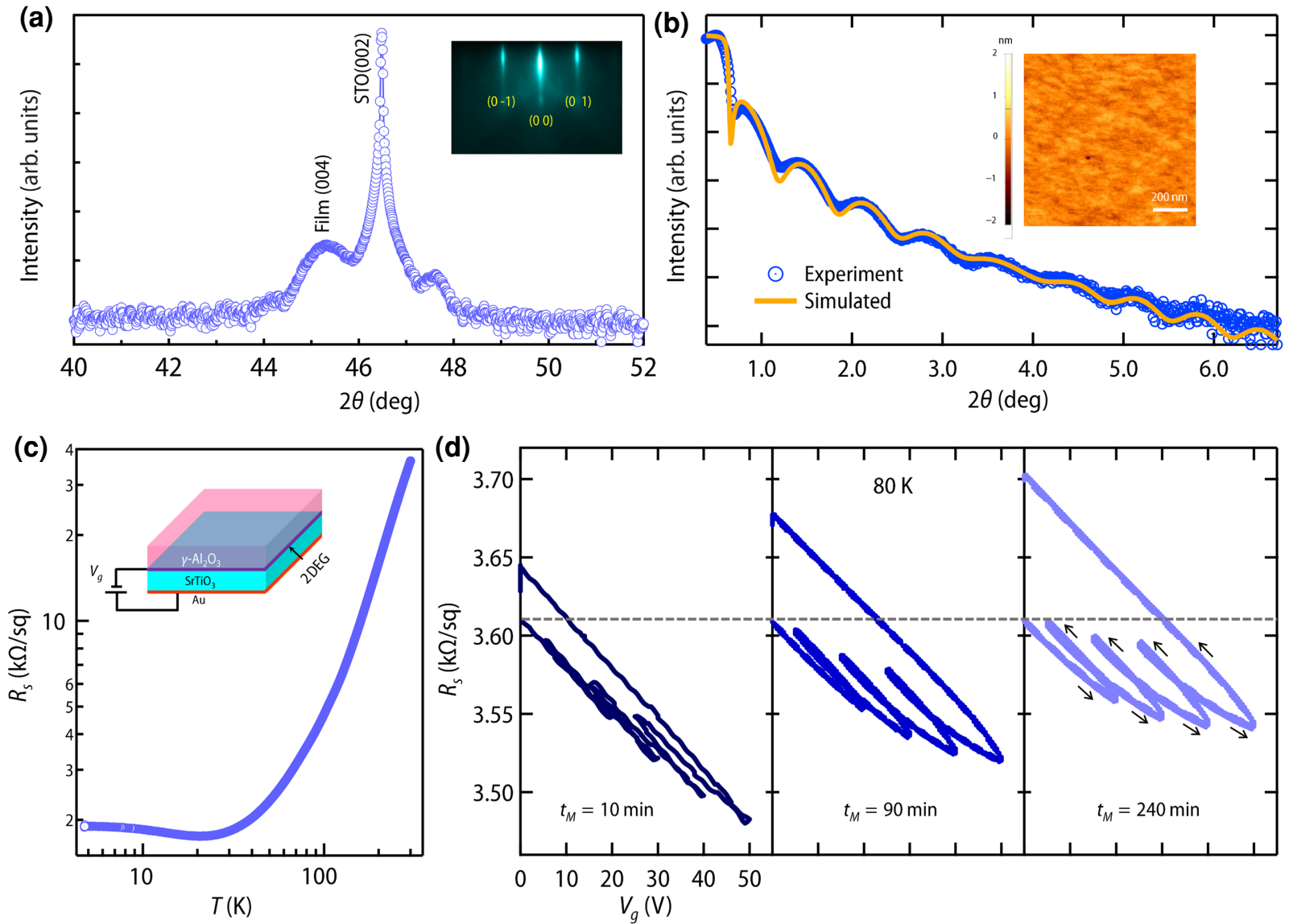

FIG. 1. (a) XRD pattern of GAO/STO heterostructure around the STO (002) peak. The inset shows a RHEED image of the GAO film taken after cooling down to room temperature. (b) X-ray reflectivity pattern of GAO film. The inset shows an AFM image of the GAO film. (c) Temperature dependence of $R_{s}$ for the GAO/STO heterostructure. The inset shows the device geometry for measurement of the resistance of the 2DEG in a back-gating configuration. (d) Variation of $R_{S}$ at $80 \mathrm{~K}$ when $V_{g}$ is swept in the sequence $0 \mathrm{~V} \rightarrow$ $20 \mathrm{~V} \rightarrow 5 \mathrm{~V} \rightarrow 30 \mathrm{~V} \rightarrow 15 \mathrm{~V} \rightarrow 40 \mathrm{~V} \rightarrow 25 \mathrm{~V} \rightarrow 50 \mathrm{~V} \rightarrow 0 \mathrm{~V}$. Total measurement time $t_{M}$ for this entire voltage sweep is 10 min, $90 \mathrm{~min}$, and $240 \mathrm{~min}$ for left, middle, and right panel, respectively. 
charge detrapping, which also disappears above $100 \mathrm{~K}$. We attribute this newly observed charge-trapping and chargedetrapping feature of carriers to the ferroelastic twin walls in STO.

\section{EXPERIMENTAL DETAILS}

A 15-unit-cell-thick GAO film is grown on an as-received mixed terminated single-crystalline STO (001) substrate $\left(5 \times 5 \times 0.5 \mathrm{~mm}^{3}\right)$ with a pulsed-laserdeposition (PLD) system. The growth is monitored by in situ high-pressure reflection high-energy electron diffraction (RHEED). The thin-film deposition is carried out at $500^{\circ} \mathrm{C}$, and the vacuum in the PLD chamber is around $10^{-6}$ Torr. A KrF excimer laser is used for the film deposition. Intense streaks of specular $(0,0)$ and off-specular $(0,-1)$ and $(0,1)$ reflections in the RHEED image [inset of Fig. 1(a)] confirm the smooth surface morphology and excellent crystallinity of the film. A Rigaku Smartlab x-ray diffractometer is used to record $\mathrm{x}$-ray reflectivity and $\mathrm{x}$ ray diffraction (XRD) patterns using $\mathrm{Cu} K_{\alpha}$ radiation. Figure 1(a) shows a $2 \theta-\omega$ XRD scan of the GAO/STO heterostructure. As expected for epitaxial growth on a STO (001) substrate, the pattern consists of a broad GAOfilm peak and a STO-substrate peak along with thickness fringes [39]. The out-of-plane lattice constant is found to be $8.01 \AA$. Atomic-force-microscopy (AFM) imaging [inset of Fig. 1(b)] further confirms the excellent surface smoothness (with a mean squared roughness of approximately $180 \mathrm{pm}$ ) of the film. Fitting of the x-ray-reflectivity data [Fig. 1(b)] using the GenX program [40] finds the thickness of the film to be $120.45 \AA$, which is close to the expected value for a 15 -unit-cell-thick film.

A 100-nm gold layer is sputtered onto the back surface of the STO substrate for back-gate experiments [Fig. 1(c)]. An ultrasonic wire bonder is used to make ohmic contacts with the interface in a van der Pauw geometry. The sheet resistance is measured in the van der Pauw geometry using a dc delta mode with a Keithley 6221 current source and a Keithley $2182 \mathrm{~A}$ nanovoltmeter. The gate voltage is applied by a Keithley 2450 source meter. The leakage current is at most $2 \mathrm{nA}$ for all the gating experiments performed in the study presented in this paper. All the electricalmeasurement meters, along with the temperature controller, have a common ground connection. Temperaturedependent electrical-resistance measurements confirm that there is a metallic interface [Fig. 1(c)] between the GAO and STO, similarly to previous reports [36,39].

\section{RESULTS AND DISCUSSION}

\section{A. Evidence of charge trapping}

We first discuss the results of gate-voltage-sweep measurements at $80 \mathrm{~K}$. Before each measurement, the sample is heated to room temperature to remove all the trapped carriers from the previous gating history. Thereafter, it is cooled down to the desired temperature, and we wait for about $6 \mathrm{~h}$ for temperature stabilization before starting any measurements. Since a positive $V_{g}$ dopes electrons into the 2DEG, the sheet resistance $\left(R_{S}\right)$ decreases when $V_{g}$ is swept from 0 to $50 \mathrm{~V}$. Surprisingly, $R_{s}$ does not follow the same path when $V_{g}$ is swept backward to $0 \mathrm{~V}$ [see Fig. 1(d)]. The offset in $R_{s}$ between the forward and the backward sweep, which has also been observed for $\mathrm{LAO} / \mathrm{STO}[22,24]$, points to the trapping of some conduction electrons from the 2DEG. Interestingly, no such offset in $R_{s}$ is observed for a negative $V_{g}$ sweep (see the Supplemental Material [41]). Furthermore, the irreversibility in the positive $V_{g}$ sweep is strongly dependent on the total time duration $\left(t_{M}\right)$ of the entire forward and backward sweep. For example, the left panel of Fig. 1(d) shows the variation of $R_{s}$ during a set of forward and backward sweeps of $V_{g}(0 \mathrm{~V} \rightarrow 20 \mathrm{~V} \rightarrow 5 \mathrm{~V} \rightarrow 30 \mathrm{~V} \rightarrow 15 \mathrm{~V}$ $\rightarrow 40 \mathrm{~V} \rightarrow 25 \mathrm{~V} \rightarrow 50 \mathrm{~V} \rightarrow 0 \mathrm{~V}$ ), performed over a duration of $10 \mathrm{~min}$. All irreversible features become more prominent with an increase in $t_{M}$ to 90 min [middle panel of Fig. 1(d)] and to 240 min [right panel of Fig. 1(d)]. Moreover, the value of $R_{s}$ at the maximum value of $V_{g}$ $(50 \mathrm{~V})$ applied decreases with an increase in $t_{M}$. On the other hand, the value of $R_{s}$ after $V_{g}$ is reduced back to zero increases with an increase in $t_{M}$. All these observations imply that the charge-trapping processes in $\mathrm{GAO} / \mathrm{STO}$ heterostructures not only depend on the applied electric field but also are strongly time dependent.

\section{B. Time-dependent measurements with positive and negative step gate voltages}

In order to understand the intriguing time dependence of charge-trapping phenomena in GAO/STO heterostructures, we design a gating protocol that consists of multiple cycles of positive and negative step gate voltages [shown in the lower panel of Fig. 2(a)] rather than a continuous sweep of $V_{g}$. The duration of each step is chosen to completely capture the concomitant trapping and detrapping processes. The upper panel of Fig. 2(a) shows the corresponding variation of $R_{s}$ with the applied $V_{g}$ at $80 \mathrm{~K}$. In the first cycle, application of a $0 \rightarrow 50 \mathrm{~V}$ step gate voltage first leads to a sudden drop in $R_{s}$ due to electrostatic charging, followed by a gradual increase in $R_{S}$ (referred to as feature A from now on), which continues for several hours. Such a slow relaxation of $R_{S}$ signifies charge trapping under a positive $V_{g}$, and understanding its microscopic origin is the primary focus of the present paper. Setting $V_{g}$ back to zero leads to a sudden jump in $R_{s}$ (similar to the discharging of a capacitor) followed by a decreasing $R_{S}$ (referred to as feature $\mathrm{B}$ for the rest of the paper). In contrast to feature $\mathrm{A}$, the detrapping feature $\mathrm{B}$ involves a much smaller change in $R_{s}$ and saturates after few hundred seconds. A further application of a negative gate voltage $(0 \mathrm{~V} \rightarrow-50 \mathrm{~V})$ leads to another fast detrapping (referred to as feature $\mathrm{C}$ ), similar 

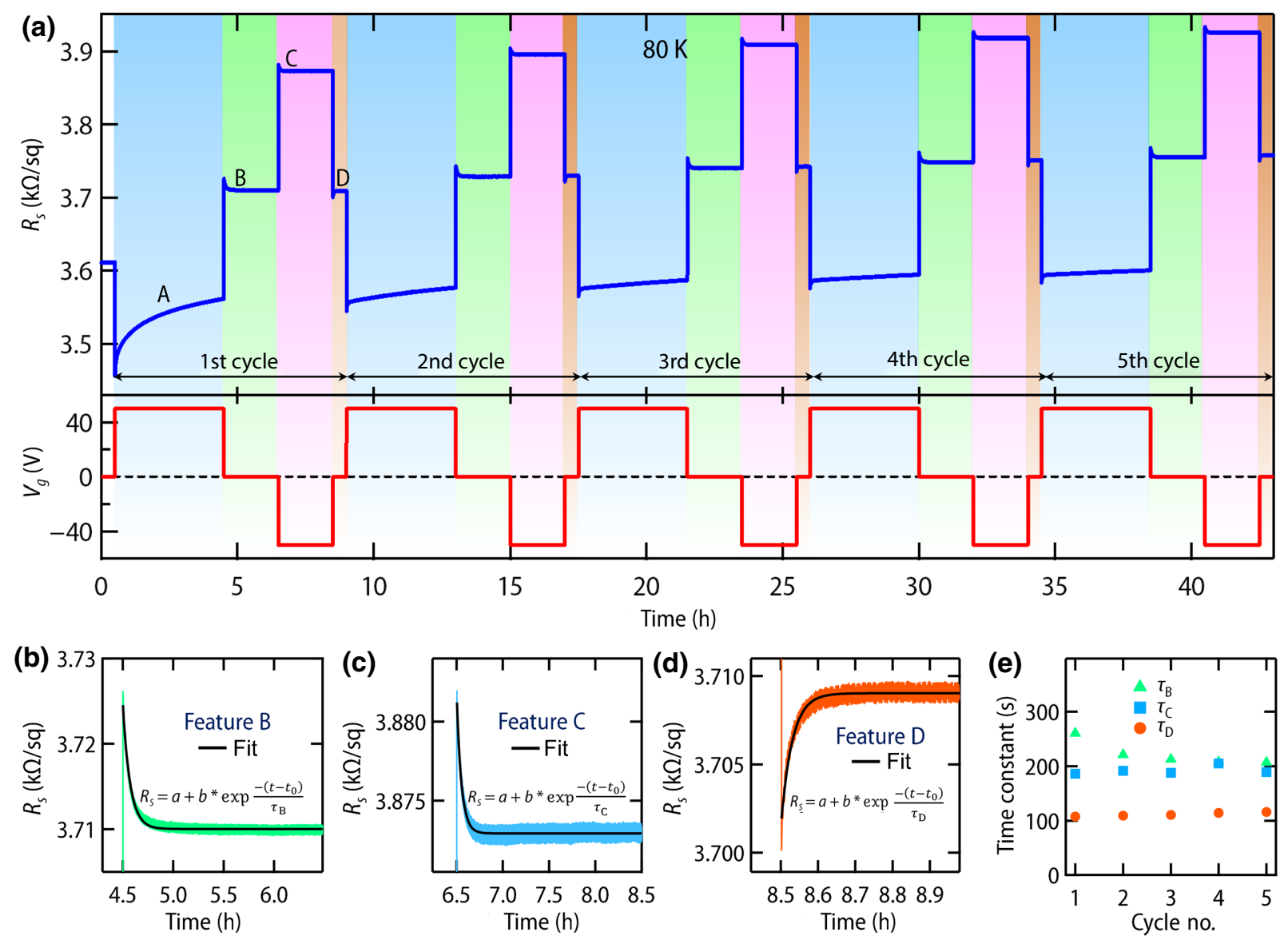

FIG. 2. (a) Back-gate-voltage sweeping protocol (lower panel) and corresponding variation of $R_{S}$ with time at $80 \mathrm{~K}$ (upper panel). (b)-(d) Magnified views of features B, C, and D along with fittings with a single exponential function. The insets of (b)-(d) show the respective functional forms of the formula used for fitting. $a$ and $b$ are constants. $t_{0}$ is the starting time of features B, C, and D. $\tau_{\mathrm{B}}, \tau_{\mathrm{C}}$, and $\tau_{\mathrm{D}}$ denote the time constants associated with features $\mathrm{B}, \mathrm{C}$, and $\mathrm{D}$, respectively. (e) Comparison of time constants of exponential features $\mathrm{B}, \mathrm{C}$, and $\mathrm{D}$ and their variation with cycle number.

to feature B. Finally, when $V_{g}$ is set to zero, $R_{s}$ drops to a value that is nearly equal to the value of $R_{s}$ at the end of feature $\mathrm{B}$, followed by the emergence of a fast-rising trapping feature, denoted $\mathrm{D}$. This entire voltage-sweeping cycle is repeated several times in succession.

While feature A has been observed earlier in the LAO/STO system [22,24], features B, C, and D are completely new. Interestingly, features B, C, and D can be fitted with a single exponential function [see Figs. 2(b)-2(d)]. Since the time constants $\tau_{B}, \tau_{C}$, and $\tau_{D}$ associated with features $\mathrm{B}, \mathrm{C}$, and $\mathrm{D}$, respectively, are similar (a few hundred seconds), the microscopic origins of these three features must be the same. Also, these time constants do not change upon multiple cycling [Fig. 2(e)]. It is important to note that feature $\mathrm{C}$ has also been independently observed by applying a step $0 \rightarrow-50 \mathrm{~V}$ (see the Supplemental Material [41]). This clearly signifies that charge trapping and detrapping under positive and negative $V_{g}$ are independent processes and are not influenced by each other. The microscopic process behind these features is discussed later in the paper.

In contrast to features $\mathrm{B}, \mathrm{C}$, and $\mathrm{D}$, feature $\mathrm{A}$ shows a strong dependence on the cycle number [Fig. 2(a)]. This is more prominent in Fig. 3(a), where we show the relative percentage change in resistance $\left[10^{2}\left(\Delta R / R_{0}\right)\right]$ at $80 \mathrm{~K}$ for different cycles. There is a drastic change from the first to the second cycle, but this quantity does not change much after that. This observation clearly emphasizes the presence of multiple simultaneous processes responsible for charge trapping under positive $V_{g}$ and, further, points to the vanishing of one of the processes after the first cycle. We cannot describe the time dependence by considering either carrier trapping due to the clustering of OVs [24] or thermal escape of carriers from a quantum well [22], or a combination of both effects (see the Supplemental Material [41]). To capture feature A entirely, we consider the following function, which comprises three simultaneous additive processes of charge trapping (see also the 
Supplemental Material [41]):

$$
R(t)=\alpha+\beta e^{-t / \tau_{\mathrm{A}}}+\zeta e^{-t / \tau_{\mathrm{OV}}}+f \ln \left(1+\frac{t}{\tau_{E}}\right) .
$$

This hybrid function provides an excellent fit to feature A for all of the cycles [Fig. 3(a)], at different temperatures (shown later) and for different values of $V_{g}$ (see also the Supplemental Material [41]). The first exponential term accounts for a purely exponential process analogous to features $\mathrm{B}, \mathrm{C}$, and D. It is demonstrated later that this contribution is related to the trapping of free carriers at ferroelastic twin walls in the STO. The second exponential term accounts for the trapping of free carriers in midgap states formed due to electric-field-induced OV clusters $[22,24,42]$. The logarithmic term represents the loss of carriers due to thermal escape of electrons from the quantum well [22], mediated by a downward band bending under positive $V_{g}$ [Fig. 3(b)]. $\alpha, \beta, \zeta$, and $f$ are constants for a given voltage and temperature. $\tau_{\mathrm{A}}$ and $\tau_{\mathrm{OV}}$ are time constants associated with two exponential processes, whereas $\tau_{E}$ is the characteristic time scale associated with thermal escape of carriers from the 2DEG.

Figure 3(c) shows the time constants (obtained from fitting) associated with the two exponential processes and with thermal escape due to band bending (see the Supplemental Material [41] for the dependence of $\beta$ and $\zeta$ on the cycle number). As is clearly evident, the time scale associated with the first exponential $\left(\tau_{\mathrm{A}}\right)$ is in the order of a few hundred seconds, and the time scale associated with the other exponential $\left(\tau_{\mathrm{OV}}\right)$ is in the order of a few hours. Interestingly $\tau_{\mathrm{A}}$ saturates at around $100 \mathrm{~s}$ after the second cycle, and this time is very similar to the time scales associated with features B, C, and D. This observation strongly points to the presence of a process analogous to that behind features $\mathrm{B}, \mathrm{C}$, and $\mathrm{D}$ contributing to charge trapping for feature $\mathrm{A}$. In contrast to $\tau_{\mathrm{A}}$, the time scale associated with the second exponential $\left(\tau_{\mathrm{OV}}\right)$ is several hours and can be attributed to the migration of OVs in the electric field followed by clustering [21,24,43-46]. OV clustering leads to the formation of midgap states, making them active centers for charge trapping. The absence of an OV-cluster contribution for negative $V_{g}$ is related to the fact that OVs move away from the interface, i.e., towards the bulk of the STO, under a negative $V_{g}$. Since the OV concentration is very small within the bulk of the STO, the probability of clustering is very low under a negative $V_{g}$ (a schematic illustration is shown in the Supplemental Material [41]). In contrast to $\tau_{\mathrm{A}}$ and $\tau_{\mathrm{OV}}$, which saturate at higher cycle numbers, $\tau_{E}$ decreases monotonically with increasing cycle number and eventually vanishes at higher cycle numbers.

The huge change in feature A from the first to the third cycle is primarily related to the dependence of the thermalescape contribution $\left\{\eta=f \ln \left[1+\left(t / \tau_{E}\right)\right]\right\}$ on the cycle
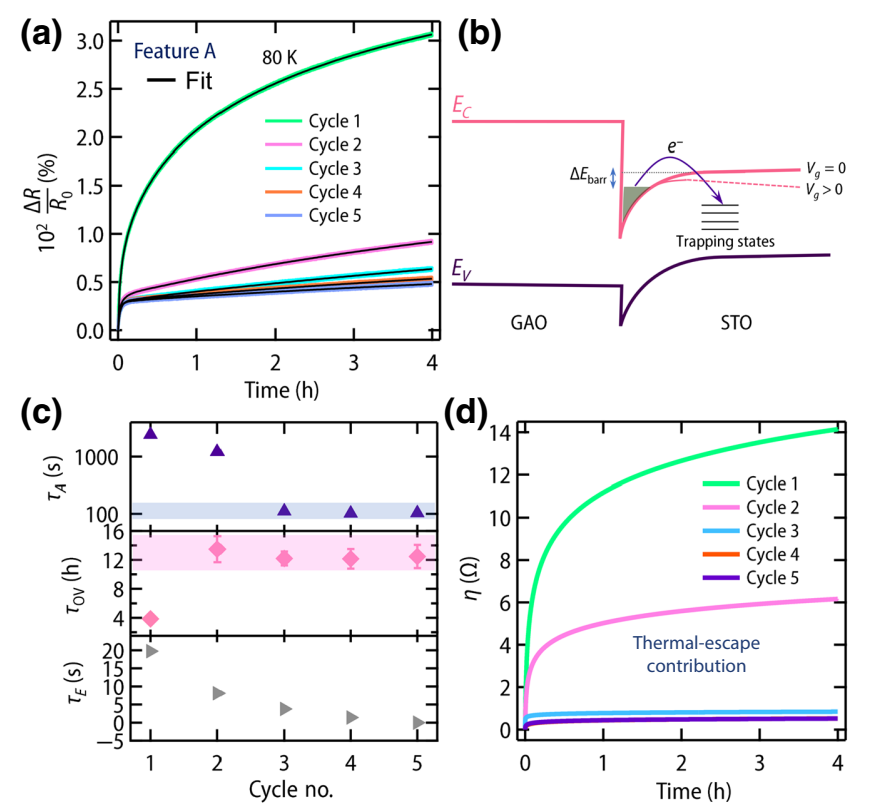

FIG. 3. (a) Relative percentage change in resistance $\left[10^{2}\left(\Delta R / R_{0}\right)\right]$ of feature $\mathrm{A}$ due to charge trapping ( $\Delta R=R-R_{0}$, where $R_{0}$ is the resistance just at the start of feature A). For comparison, the starting times of feature A for all the cycles have been manually shifted to zero. (b) Schematic illustration to show thermal escape of electrons under positive $V_{g}$. Application of a positive back-gate voltage bends the conduction band and lowers the barrier height $\Delta E_{\text {barr }}$ for thermal escape. Application of a negative $V_{g}$ would bend the band upwards in energy, which would increase $\Delta E_{\text {barr }}$ for thermal escape. (c) Variation of $\tau_{\mathrm{A}}, \tau_{\mathrm{OV}}$, and $\tau_{E}$ upon multiple cycling. (d) Variation of thermal-escape contribution with increasing cycle number.

number, as shown in Fig. 3(d) (see also the Supplemental Material [41]). $\eta$ becomes negligible after the second cycle. This is expected, as thermal escape is controlled by the energy barrier $\Delta E_{\text {barr }}$ [Fig. 3(b)]. Once the charges have escaped irreversibly from the quantum well in the first cycle, the Fermi level goes deeper into the well [Fig. 3(b)]. This increases $\Delta E_{\text {barr }}$, and therefore lowers the probability of thermal escape in subsequent cycles. The thermal-escape contribution is also absent for a negative gate voltage, as $\Delta E_{\text {barr }}$ increases due to band bending towards higher energy under negative $V_{g}$ [Fig. 3(b)].

\section{Charge trapping in twin walls in $\mathrm{SrTiO}_{3}$}

In order to check how these charge-trapping and chargedetrapping features evolve across the ferroelastic transition of STO, we carry out similar measurements at several temperatures. We first discuss the variation of feature $\mathrm{B}$, which is captured by a single exponential function at $80 \mathrm{~K}$. As evident from Fig. 4(a) (first cycle of feature B), this feature gradually decreases with an increase in temperature, and vanishes around $100 \mathrm{~K}$, which is very close to the 

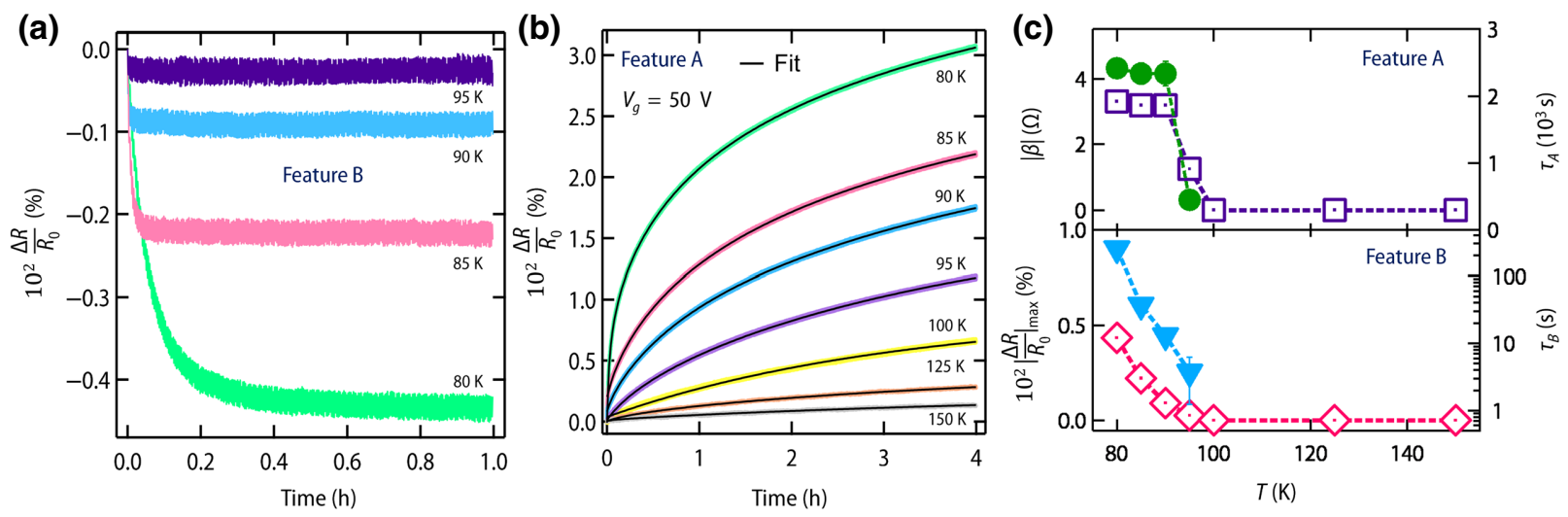

FIG. 4. (a) Temperature dependence of feature B (first cycle). (b) Temperature dependence of feature A (first cycle) for $V_{g}=50 \mathrm{~V}$. (c) Temperature evolution of $\tau_{\mathrm{A}}$ and $|\beta|$ obtained from fitting of feature A (upper panel). The lower panel shows the temperature evolution of $\tau_{\mathrm{B}}$ obtained from fitting of feature $\mathrm{B}$, and the magnitude of the maximum relative percentage change in resistance $\left(10^{2}\left|\Delta R / R_{0}\right|_{\max }\right)$ due to detrapping in feature $\mathrm{B}$. The left and right axes in upper and lower panels of (c) correspond to open and filled symbols, respectively.

ferroelastic phase transition temperature of STO. A similar temperature dependence is also observed for features C and D (see the Supplemental Material [41]). Feature A also gradually decreases with $T$ and becomes negligibly small above $150 \mathrm{~K}$ [Fig. 4(b)]. An analysis of this feature using Eq. (1) reveals that both the thermal-escape $(\eta)$ and the OV-cluster $\left(\zeta e^{-t / \tau_{\mathrm{OV}}}\right)$ contribution terms survive up to $150 \mathrm{~K}$ (see the Supplemental Material [41]). However, the contribution of the $\beta e^{-t / \tau_{\mathrm{A}}}$ term to feature $\mathrm{A}$ vanishes around $100 \mathrm{~K}$, similarly to features $\mathrm{B}, \mathrm{C}$, and $\mathrm{D}$. This is more prominent in Fig. 4(c), where we plot $\tau_{\mathrm{A}}$ and $|\beta|$ obtained from the fitting of feature A (upper panel) along with $\tau_{\mathrm{B}}$ and $10^{2}\left|\Delta R / R_{0}\right|_{\max }$ for feature $\mathrm{B}$ (lower panel). $|\beta|$ obtained from feature $\mathrm{A}$ and $10^{2}\left|\Delta R / R_{0}\right|_{\max }$ for feature $\mathrm{B}$ in the second and third cycles of measurements also vanish at $100 \mathrm{~K}$ (see the Supplemental Material [41]).

Based on this experimental evidence, we propose that the following microscopic processes below $100 \mathrm{~K}$ are responsible for the additional charge-trapping contribution to feature A and are the origin of features B, C, and D. As mentioned earlier, polar twin walls, which appear spontaneously below the ferroelastic transition temperature of STO, can trap mobile electrons even in the absence of any electric field [first panel of Fig. 5(a)]. Switching on the electric field can affect the amount of trapped charge at the twin walls in two ways: it can lead to the formation of new twin walls [35] and/or change the polarity of the twin walls. In the first scenario, newly formed twin walls would become an additional source of trapping and could be the origin of the additional charge-trapping contribution to feature A below $100 \mathrm{~K}$. However, we rule out such a possibility in the present case for the following reasons.

The existence of a certain cutoff field for the formation of new twin walls was reported earlier in a scanning-electron-microscopy study [35]. A more recent polarized-optical-microscopy study [47] demonstrated further that even an electric field as high as $4 \mathrm{kV} / \mathrm{cm}$ is not sufficient either to move twin walls or to form new twin walls above $40 \mathrm{~K}$ (which is much lower than the range of temperatures investigated in the present study). To check whether there is any cutoff field for an additional charge-trapping contribution to feature A in our system, we plot the coefficient $|\beta|$ as a function of the maximum value of the step voltage $\left(V_{g}^{\max }\right)$ in Fig. $5(\mathrm{~b})$ at $80 \mathrm{~K}$ (see the Supplemental Material [41] for the $V_{g}$ dependence of the thermal-escape and OV-cluster contributions to charge trapping under positive $V_{g}$ ). As evident, there is no cutoff voltage for the appearance of this additional contribution. This observation further strengthens our claim that the additional charge-trapping contribution to feature A below $T_{c}$ of STO cannot be attributed to electric-field-induced formation of new twin walls.

Interestingly, the coefficient $|\beta|$ scales almost linearly with the maximum value of $V_{g}\left(V_{g}^{\max }\right)$ for applied positive step voltages up to $50 \mathrm{~V}$. At higher $V_{g}$, this dependence deviates from a linear behavior (see the Supplemental Material [41]). We also note that a previous scanningstress-microscopy study demonstrated that the polarity of a twin wall changed linearly with the applied stress at the twin wall [30]. We believe that, due to coupling between the ferroelastic and ferroelectric order parameters in STO [48], the same linear dependence is reflected in our electric-field-dependent measurement. This would mean that, upon application of a positive $V_{g}$, there is an increase in the polarity of the twin walls. This would enhance the charge-holding capacity of individual twin walls $[33,49]$ and lead to an additional charge-trapping contribution $\left[\beta e^{-t / \tau_{\mathrm{A}}}\right.$, referred to as the twin-wall contribution (TWC) from now on] to feature A below $100 \mathrm{~K}$ [second panel of Fig. 5(a)]. 
(a)
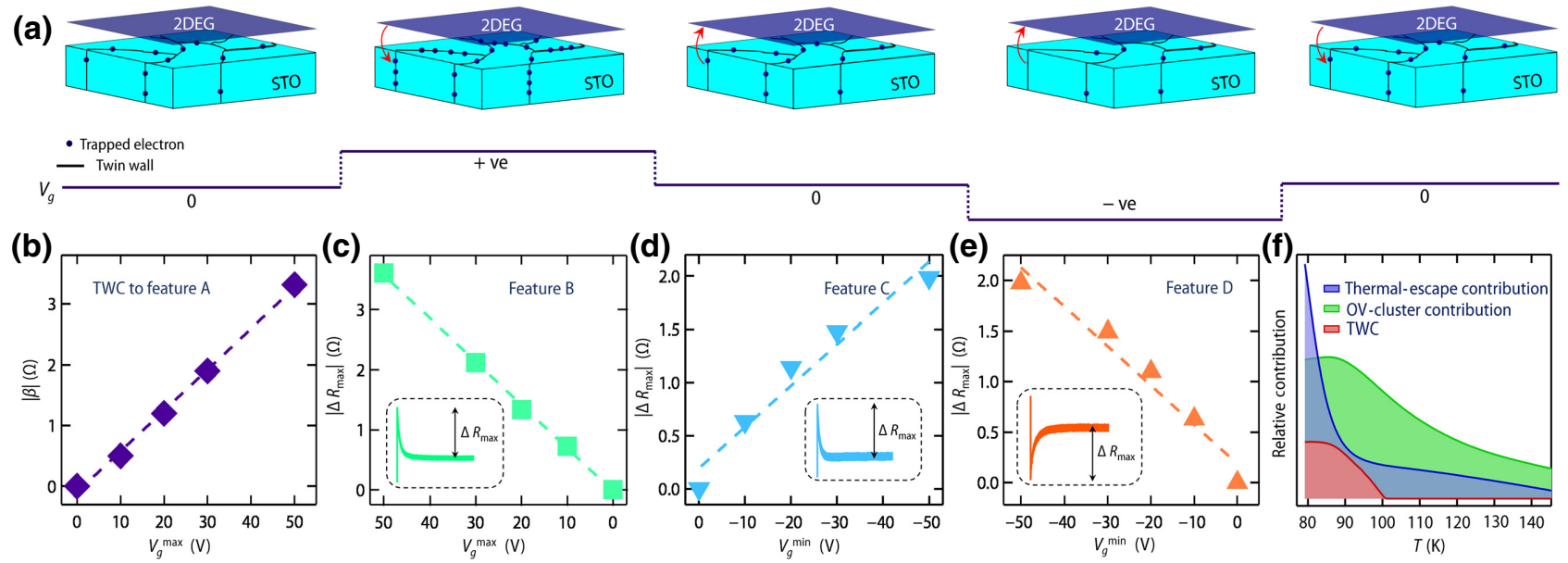

(c)
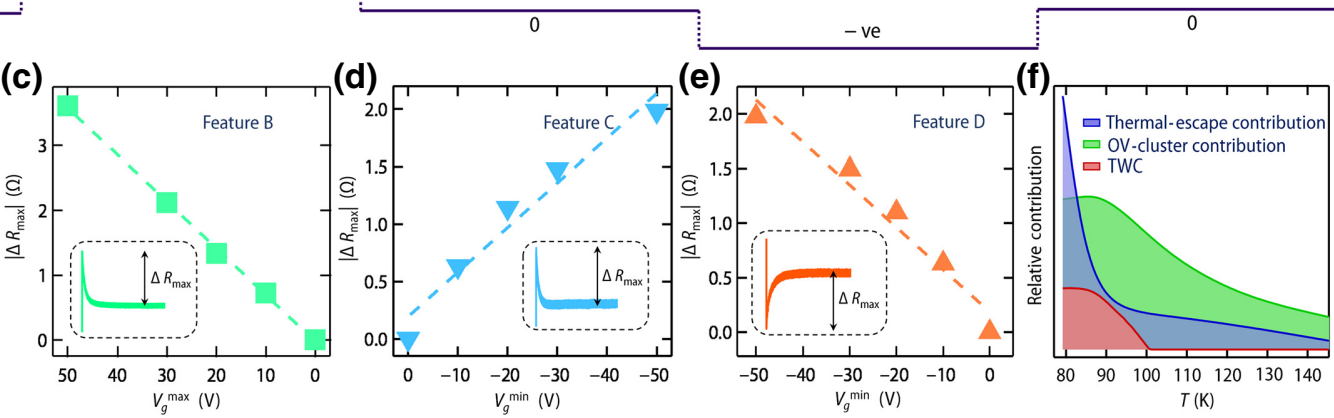

FIG. 5. (a) A set of schematic illustrations to show electric-field-induced trapping and detrapping of charges at twin walls in STO. The first panel shows trapped electrons at twin walls in STO at zero back-gate voltage. Application of a $0 \rightarrow$ positive step $V_{g}$ leads to transfer of electrons from the 2DEG to the twin walls (second panel). Switching off the field after the $0 \rightarrow$ positive step $V_{g}$ leads to reversible transfer of electrons from the twin walls to the 2DEG (third panel). Application of a $0 \rightarrow$ negative step $V_{g}$ leads to transfer of electrons from the twin walls to the 2DEG (fourth panel). Switching off the voltage after application of the $0 \rightarrow$ negative step $V_{g}$ leads to reversible transfer of electrons from the 2DEG to the twin walls (fifth panel). For visual clarity, the STO substrate and the 2DEG are shown physically separated from each other. (b) Variation of twin-wall contribution to feature A in the first cycle at $80 \mathrm{~K}$ with $V_{g}^{\max }$. (c) Variation of magnitude of maximum change in resistance due to the detrapping feature B in the first cycle at $80 \mathrm{~K}$ with $V_{g}^{\max }$. (d) Variation of magnitude of maximum change in resistance due to feature $\mathrm{C}$ in the first cycle at $80 \mathrm{~K}$ with $V_{g}^{\min }$. (e) Variation of magnitude of maximum change in resistance due to feature $\mathrm{D}$ in the first cycle at $80 \mathrm{~K}$ with $V_{g}^{\min }$. (f) Schematic illustration depicting the different charge-trapping mechanisms present above and below $T_{c}$ of STO. The change in resistance due to each of the three processes at the end of feature A (i.e., $4 \mathrm{~h}$ after the application of a constant $V_{g}=50 \mathrm{~V}$ ) is used to quantify their relative contributions. The dashed lines in (b)-(e) denote fittings with a linear function. The insets of (c)-(e) show the definitions of $\Delta R_{\max }$ for the respective features.

Removal of the positive electric field leads to the reversible transfer of electrons from the charged twin walls to the 2DEG [third panel of Fig. 5(a)]. This pumps a few extra carriers into the 2DEG, leading to the decaying nature of feature B. Similarly to the TWC to feature A, the magnitude of the maximum change in resistance due to the detrapping feature $\mathrm{B}\left(\left|\Delta R_{\max }\right|=\left|R_{\text {sat }}-R_{0}\right|\right.$, where $R_{\text {sat }}$ is the saturation value of the resistance and $R_{0}$ is the resistance at a manually shifted zero time) [inset of Fig. 5(c)] also shows the same linear dependence on the positive voltage applied in the previous step $\left(V_{g}^{\max }\right)$ up to $50 \mathrm{~V}$ [Fig. 5(c)]. Interestingly, the value of $|\beta|$ obtained from the fitting of feature $\mathrm{A}$ is very close to $\left|\Delta R_{\max }\right|$ for feature $\mathrm{B}$ at each $V_{g}$. This observation signifies that the charge trapping at twin walls in STO under a positive $V_{g}$ is a completely reversible process (see also the Supplemental Material [41]). This is in sharp contrast to the completely irreversible nature of the thermal-escape and OV-clustering contributions to charge trapping under positive $V_{g}$.

Application of a negative $V_{g}$ further transfers electrons from the twin walls to the 2DEG [fourth panel of Fig. 5(a)], resulting in the decaying feature $\mathrm{C}$. The absence of any cutoff field and the almost linear dependence of $\left|\Delta R_{\max }\right|$ [see the inset of Fig. 5(d) for the definition of $\Delta R_{\max }$ ] on the minimum value of $V_{g}\left(V_{g}^{\mathrm{min}}\right)$ for a negative step voltage [Fig. 5(d)] indicates that the decreased polarity of the twin walls under a negative $V_{g}$ is the microscopic origin of feature C. Switching off this negative voltage thereafter leads to a reversible transfer of electrons back to the twin walls [fifth panel of Fig. 5(a)] and results in the appearance of the rising feature D. $\left|\Delta R_{\max }\right|$ [see the inset of Fig. 5(e) for the definition of $\Delta R_{\max }$ ] for feature $\mathrm{D}$ also scales almost linearly with $V_{g}^{\text {min }}$ [Fig. 5(e)]. We also note that the values of $\left|\Delta R_{\max }\right|$ for features $\mathrm{C}$ and $\mathrm{D}$ are very similar, as expected for a reversible trapping and detrapping process. However, $\left|\Delta R_{\max }\right|$ for feature $\mathrm{C}$ is lower than the corresponding $|\beta|$, for each $V_{g}$. This peculiar behavior can be attributed to the multiorbital nature of the electronic bands near the Fermi level [50].

In the study presented in this paper, we investigate different charge-trapping and charge-detrapping mechanisms across the structural phase transition temperature of STO. It is also important to note that the polar twin walls in STO undergo a series of phase transitions from a domain glass phase to domain solid phase at lower temperatures [51]. We expect similar glassy behavior to be reflected in the temporal relaxation of the resistance (under an applied electric field) in our samples at lower temperatures. 


\section{CONCLUSIONS}

In conclusion, our detailed temperature- and electricfield-dependent back-gating experiments on a $\mathrm{GAO} / \mathrm{STO}$ heterostructure reveal that the charge trapping in $\mathrm{GAO} / \mathrm{STO}$ is quite complex and requires invoking simultaneous multiple trapping mechanisms [Fig. 5(f)]. We detect a clear signature of charge trapping (detrapping) at (from) ferroelastic twin walls in the STO substrate below its structural transition temperature. Such charge trapping and detrapping at twin walls have not been reported for any $\mathrm{SrTiO}_{3}$-based heterostructures so far. This contribution, for $\mathrm{GAO} / \mathrm{STO}$ heterostructures, is completely reversible with cycling between positive and negative $V_{g}$. In contrast, the charge-trapping contributions from the thermal escape of electrons from the quantum well and the formation of midgap states due to oxygen-vacancy clustering are completely irreversible and are present only under positive gate voltages.

Our study highlights that these charge-trapping and charge-detrapping processes could introduce timedependent features into electrostatic-gating experiments on devices involving STO as a dielectric material and that proper care must be taken in interpreting any data, especially in the case of studies of memory effects. The present study also suggests that the scattering of conduction electrons at domain walls in STO could be another limiting factor for the electron mobility of STO-based 2DEGs [52,53]. Further, these twin walls can be treated as nanoscale local gates, as the local carrier density can be changed by tuning the polarity. Since these twin walls can also be moved spatially by the application of stress [30] and/or an electric field [47], they could be used to build memory elements $[54,55]$ where polar twin walls would carry information similarly to the racetrack memory technology [56].

\section{ACKNOWLEDGMENTS}

This work was funded by a DST Nanomission grant (Grant No. DST/NM/NS/2018/246) and a SERB Early Career Research Award (Grant No. ECR/2018/001512). S.M. also acknowledges support from the Infosys Foundation, Bangalore. The authors acknowledge the AFM and XRD facilities at the Department of Physics, IISc Bangalore.

[1] C. Ahn, J.-M. Triscone, and J. Mannhart, Electric field effect in correlated oxide systems, Nature 424, 1015 (2003).

[2] W. G. Vandenberghe and M. V. Fischetti, Imperfect twodimensional topological insulator field-effect transistors, Nat. Commun. 8, 1 (2017).

[3] J. L. Collins, A. Tadich, W. Wu, L. C. Gomes, J. N. Rodrigues, C. Liu, J. Hellerstedt, H. Ryu, S. Tang,
S.-K. Mo, et al., Electric-field-tuned topological phase transition in ultrathin $\mathrm{Na}_{3} \mathrm{Bi}$, Nature 564, 390 (2018).

[4] A. Ohtomo and H. Hwang, A high-mobility electron gas at the $\mathrm{LaAlO}_{3} / \mathrm{SrTiO}_{3}$ heterointerface, Nature 427, 423 (2004).

[5] J. Mannhart and D. G. Schlom, Oxide interfaces - an opportunity for electronics, Science 327, 1607 (2010).

[6] J. Mannhart, D. H. Blank, H. Hwang, A. Millis, and J.-M. Triscone, Two-dimensional electron gases at oxide interfaces, MRS Bull. 33, 1027 (2008).

[7] J.-S. Lee, Y. Xie, H. Sato, C. Bell, Y. Hikita, H. Hwang, and C.-C. Kao, Titanium $d_{x y}$ ferromagnetism at the $\mathrm{LaAlO}_{3} / \mathrm{SrTiO}_{3}$ interface, Nat. Mater. 12, 703 (2013).

[8] A. Brinkman, M. Huijben, M. Van Zalk, J. Huijben, U. Zeitler, J. Maan, W. G. van der Wiel, G. Rijnders, D. H. Blank, and H. Hilgenkamp, Magnetic effects at the interface between non-magnetic oxides, Nat. Mater. 6, 493 (2007).

[9] N. Reyren, S. Thiel, A. Caviglia, L. F. Kourkoutis, G. Hammerl, C. Richter, C. W. Schneider, T. Kopp, A.-S. Rüetschi, and D. Jaccard, et al., Superconducting interfaces between insulating oxides, Science 317, 1196 (2007).

[10] S. Stemmer and S. James Allen, Two-dimensional electron gases at complex oxide interfaces, Annu. Rev. Mater. Res. 44, 151 (2014).

[11] P. King, S. M. Walker, A. Tamai, A. De La Torre, T. Eknapakul, P. Buaphet, S.-K. Mo, W. Meevasana, M. Bahramy, and F. Baumberger, Quasiparticle dynamics and spin-orbital texture of the $\mathrm{SrTiO}_{3}$ two-dimensional electron gas, Nat. Commun. 5, 1 (2014).

[12] D. C. Vaz, P. Noël, A. Johansson, B. Göbel, F. Y. Bruno, G. Singh, S. Mckeown-Walker, F. Trier, L. M. VicenteArche, A. Sander, et al., Mapping spin-charge conversion to the band structure in a topological oxide two-dimensional electron gas, Nat. Mater. 18, 1187 (2019).

[13] S. K. Ojha, S. K. Gogoi, M. M. Patidar, R. K. Patel, P. Mandal, S. Kumar, R. Venkatesh, V. Ganesan, M. Jain, and S. Middey, Oxygen vacancy-induced topological Hall effect in a nonmagnetic band insulator, Adv. Quantum Technol. 3, 2000021 (2020).

[14] S. Thiel, G. Hammerl, A. Schmehl, C. W. Schneider, and J. Mannhart, Tunable quasi-two-dimensional electron gases in oxide heterostructures, Science 313, 1942 (2006).

[15] A. Caviglia, S. Gariglio, N. Reyren, D. Jaccard, T. Schneider, M. Gabay, S. Thiel, G. Hammerl, J. Mannhart, and J.-M. Triscone, Electric field control of the $\mathrm{LaAlO}_{3} / \mathrm{SrTiO}_{3}$ interface ground state, Nature 456, 624 (2008).

[16] A. Caviglia, M. Gabay, S. Gariglio, N. Reyren, C. Cancellieri, and J.-M. Triscone, Tunable Rashba Spin-Orbit Interaction at Oxide Interfaces, Phys. Rev. Lett. 104, 126803 (2010).

[17] F. Bi, M. Huang, S. Ryu, H. Lee, C.-W. Bark, C.-B. Eom, P. Irvin, and J. Levy, Room-temperature electronicallycontrolled ferromagnetism at the $\mathrm{LaAlO}_{3} / \mathrm{SrTiO}_{3}$ interface, Nat. Commun. 5, 1 (2014).

[18] H. Weaver, Dielectric properties of single crystals of $\mathrm{SrTio}_{3}$ at low temperatures, J. Phys. Chem. Solids 11, 274 (1959).

[19] R. C. Neville, B. Hoeneisen, and C. A. Mead, Permittivity of strontium titanate, J. Appl. Phys. 43, 2124 (1972). 
[20] R. Viana, P. Lunkenheimer, J. Hemberger, R. Böhmer, and A. Loidl, Dielectric spectroscopy in $\mathrm{SrTio}_{3}$, Phys. Rev. B 50, 601 (1994).

[21] D. V. Christensen, F. Trier, M. von Soosten, G. E. D. K. Prawiroatmodjo, T. S. Jespersen, Y. Z. Chen, and N. Pryds, Electric field control of the $\gamma-\mathrm{Al}_{2} \mathrm{O}_{3} / \mathrm{SrTiO}_{3}$ interface conductivity at room temperature, Appl. Phys. Lett. 109, 021602 (2016).

[22] J. Biscaras, S. Hurand, C. Feuillet-Palma, A. Rastogi, R. Budhani, N. Reyren, E. Lesne, J. Lesueur, and N. Bergeal, Limit of the electrostatic doping in twodimensional electron gases of $\mathrm{LaXo}_{3}(x=\mathrm{Al}, \mathrm{Ti}) / \mathrm{SrTio}_{3}$, Sci. Rep. 4, 6788 (2014).

[23] W. Liu, S. Gariglio, A. Fête, D. Li, M. Boselli, D. Stornaiuolo, and J.-M. Triscone, Magneto-transport study of top-and back-gated $\mathrm{LaAlO}_{3} / \mathrm{SrTiO}_{3}$ heterostructures, APL Mater. 3, 062805 (2015).

[24] C. Yin, A. E. Smink, I. Leermakers, L. M. Tang, N. Lebedev, U. Zeitler, W. G. van der Wiel, H. Hilgenkamp, and J. Aarts, Electron Trapping Mechanism in $\mathrm{LaAlO}_{3} / \mathrm{SrTiO}_{3}$ Heterostructures, Phys. Rev. Lett. 124, 017702 (2020).

[25] V. V. Bal, Z. Huang, K. Han, Ariando, T. Venkatesan, and $\mathrm{V}$. Chandrasekhar, Electrostatic tuning of magnetism at the conducting (111) $\left(\mathrm{La}_{0.3} \mathrm{Sr}_{0.7}\right)\left(\mathrm{Al}_{0.65} \mathrm{Ta}_{0.35}\right) / \mathrm{SrTiO}_{3}$ interface, Appl. Phys. Lett. 111, 081604 (2017).

[26] C.-P. Chang, M.-W. Chu, H. T. Jeng, S.-L. Cheng, J. G. Lin, J.-R. Yang, and C. H. Chen, Condensation of two-dimensional oxide-interfacial charges into onedimensional electron chains by the misfit-dislocation strain field, Nat. Commun. 5, 3522 (2014).

[27] F. Gunkel, D. V. Christensen, Y. Z. Chen, and N. Pryds, Oxygen vacancies: The (in)visible friend of oxide electronics, Appl. Phys. Lett. 116, 120505 (2020).

[28] R. A. Cowley, Lattice dynamics and phase transitions of strontium titanate, Phys. Rev. 134, A981 (1964).

[29] A. Schiaffino and M. Stengel, Macroscopic Polarization from Antiferrodistortive Cycloids in Ferroelastic $\mathrm{SrTiO}_{3}$, Phys. Rev. Lett. 119, 137601 (2017).

[30] Y. Frenkel, N. Haham, Y. Shperber, C. Bell, Y. Xie, Z. Chen, Y. Hikita, H. Y. Hwang, E. K. H. Salje, and B. Kalisky, Imaging and tuning polarity at $\mathrm{SrTiO}_{3}$ domain walls, Nat. Mater. 16, 1203 (2017).

[31] E. K. H. Salje, O. Aktas, M. A. Carpenter, V. V. Laguta, and J. F. Scott, Domains Within Domains and Walls Within Walls: Evidence for Polar Domains in Cryogenic $\mathrm{SrTiO}_{3}$, Phys. Rev. Lett. 111, 247603 (2013).

[32] P. Zubko, G. Catalan, A. Buckley, P. R. L. Welche, and J. F. Scott, Strain-Gradient-Induced Polarization in $\mathrm{SrTiO}_{3}$ Single Crystals, Phys. Rev. Lett. 99, 167601 (2007).

[33] B. Kalisky, E. M. Spanton, H. Noad, J. R. Kirtley, K. C. Nowack, C. Bell, H. K. Sato, M. Hosoda, Y. Xie, Y. Hikita, C. Woltmann, G. Pfanzelt, R. Jany, C. Richter, H. Y. Hwang, J. Mannhart, and K. A. Moler, Locally enhanced conductivity due to the tetragonal domain structure in $\mathrm{LaAlO}_{3} / \mathrm{SrTiO}_{3}$ heterointerfaces, Nat. Mater. 12, 1091 (2013).

[34] M. Honig, J. A. Sulpizio, J. Drori, A. Joshua, E. Zeldov, and S. Ilani, Local electrostatic imaging of striped domain order in $\mathrm{LaAlO}_{3} / \mathrm{SrTiO}_{3}$, Nat. Mater. 12, 1112 (2013).
[35] H. J. H. Ma, S. Scharinger, S. W. Zeng, D. Kohlberger, M. Lange, A. Stöhr, X. R. Wang, T. Venkatesan, R. Kleiner, J. F. Scott, J. M. D. Coey, D. Koelle, Ariando, Local Electrical Imaging of Tetragonal Domains and Field-Induced Ferroelectric Twin Walls in Conducting $\mathrm{SrTiO}_{3}$, Phys. Rev. Lett. 116, 257601 (2016).

[36] Y. Chen, N. Bovet, F. Trier, D. Christensen, F. Qu, N. H. Andersen, T. Kasama, W. Zhang, R. Giraud, J. Dufouleur, and et al., A high-mobility two-dimensional electron gas at the spinel/perovskite interface of $\gamma-\mathrm{Al}_{2} \mathrm{O}_{3} / \mathrm{SrTiO}_{3}$, Nat. Commun. 4, 1 (2013).

[37] P. Schütz, D. V. Christensen, V. Borisov, F. Pfaff, P. Scheiderer, L. Dudy, M. Zapf, J. Gabel, Y. Chen, N. Pryds, and et al., Microscopic origin of the mobility enhancement at a spinel/perovskite oxide heterointerface revealed by photoemission spectroscopy, Phys. Rev. B 96, 161409 (2017).

[38] D. V. Christensen, M. von Soosten, F. Trier, T. S. Jespersen, A. Smith, Y. Chen, and N. Pryds, Controlling the carrier density of $\mathrm{SrTiO}_{3}$-based heterostructures with annealing, Adv. Electron. Mater. 3, 1700026 (2017).

[39] Y. Cao, X. Liu, P. Shafer, S. Middey, D. Meyers, M. Kareev, Z. Zhong, J.-W. Kim, P. J. Ryan, E. Arenholz, and J. Chakhalian, Anomalous orbital structure in a spinel-perovskite interface, npj Quantum Mater. 1, 16009 (2016).

[40] M. Björck and G. Andersson, GenX: An extensible X-ray reflectivity refinement program utilizing differential evolution, J. Appl. Crystallogr. 40, 1174 (2007).

[41] See Supplemental Material at http://link.aps.org/supple mental/10.1103/PhysRevApplied.15.054008 for $R_{s}$ during a negative $V_{g}$ sweep, independent observation of feature $\mathrm{C}$, the functional form of feature $\mathrm{A}$, the dependence of the $\mathrm{OV}$ and twin-wall contributions to feature A on the cycle number, a schematic illustration to explain the electricfield-induced clustering of OVs and their redistribution during a complete voltage cycle, the temperature dependence of feature $\mathrm{A}$, the temperature dependence of features $\mathrm{C}$ and $\mathrm{D}$, the temperature dependence of the twin-wall contribution to features $\mathrm{A}$ and $\mathrm{B}$ in the second and third cycle, the reversible nature of the twin-wall contribution to feature A under positive $V_{g}$ and to charge detrapping under negative $V_{g}$, the effect of the electric field strength on charge trapping under positive $V_{g}$, and additional data on another 15-unit-cell $\gamma-\mathrm{Al}_{2} \mathrm{O}_{3} / \mathrm{SrTiO}_{3}$ sample. The Supplemental Material also includes Ref. [57].

[42] S. Seri, M. Schultz, and L. Klein, Thermally activated recovery of electrical conductivity in $\mathrm{LaAlO}_{3} / \mathrm{SrTiO}_{3}$, Phys. Rev. B 87, 125110 (2013).

[43] Y. Lei, Y. Li, Y. Z. Chen, Y. W. Xie, Y. S. Chen, S. H. Wang, J. Wang, B. G. Shen, N. Pryds, H. Y. Hwang, and J. R. Sun, Visible-light-enhanced gating effect at the $\mathrm{LaAlO}_{3} / \mathrm{SrTiO}_{3}$ interface, Nat. Commun. 5, 5554 (2014).

[44] K. Szot, W. Speier, G. Bihlmayer, and R. Waser, Switching the electrical resistance of individual dislocations in singlecrystalline $\mathrm{SrTiO}_{3}$, Nat. Mater. 5, 312 (2006).

[45] R. A. De Souza, V. Metlenko, D. Park, and T. E. Weirich, Behavior of oxygen vacancies in single-crystal $\mathrm{SrTiO}_{3}$ : Equilibrium distribution and diffusion kinetics, Phys. Rev. B 85, 174109 (2012). 
[46] J. Hanzig, M. Zschornak, F. Hanzig, E. Mehner, H. Stöcker, B. Abendroth, C. Röder, A. Talkenberger, G. Schreiber, D. Rafaja, S. Gemming, and D. C. Meyer, Migrationinduced field-stabilized polar phase in strontium titanate single crystals at room temperature, Phys. Rev. B 88, 024104 (2013).

[47] B. Casals, S. van Dijken, G. Herranz, and E. K. H. Salje, Electric-field-induced avalanches and glassiness of mobile ferroelastic twin domains in cryogenic $\mathrm{SrTiO}_{3}$, Phys. Rev. Res. 1, 032025 (2019).

[48] D. Pesquera, M. A. Carpenter, and E. K. H. Salje, Glasslike Dynamics of Polar Domain Walls in Cryogenic $\mathrm{SrTiO}_{3}$, Phys. Rev. Lett. 121, 235701 (2018).

[49] P. Mokrý, A. K. Tagantsev, and J. Fousek, Pressure on charged domain walls and additional imprint mechanism in ferroelectrics, Phys. Rev. B 75, 094110 (2007).

[50] F. Zhang, P. Lv, Y. Zhang, S. Huang, C.-M. Wong, H.-M. Yau, X. Chen, Z. Wen, X. Jiang, C. Zeng, J. Hong, and J.-y. Dai, Modulating the Electrical Transport in the TwoDimensional Electron Gas at $\mathrm{LaAlO}_{3} / \mathrm{SrTiO}_{3}$ Heterostructures by Interfacial Flexoelectricity, Phys. Rev. Lett. 122, 257601 (2019).
[51] S. Kustov, I. Liubimova, and E. K. H. Salje, Domain Dynamics in Quantum-Paraelectric $\mathrm{SrTiO}_{3}$, Phys. Rev. Lett. 124, 016801 (2020).

[52] F. Trier, D. V. Christensen, and N. Pryds, Electron mobility in oxide heterostructures, J. Phys. D: Appl. Phys. 51, 293002 (2018).

[53] D. V. Christensen, Y. Frenkel, P. Schütz, F. Trier, S. Wissberg, R. Claessen, B. Kalisky, A. Smith, Y. Z. Chen, and N. Pryds, Electron Mobility in $\gamma-\mathrm{Al}_{2} \mathrm{O}_{3} / \mathrm{SrTiO}_{3}$, Phys. Rev. Appl. 9, 054004 (2018).

[54] E. K. H. Salje, Ferroelastic domain walls as templates for multiferroic devices, J. Appl. Phys. 128, 164104 (2020).

[55] G. F. Nataf, M. Guennou, J. M. Gregg, D. Meier, J. Hlinka, E. K. H. Salje, and J. Kreisel, Domain-wall engineering and topological defects in ferroelectric and ferroelastic materials, Nat. Rev. Phys. 2, 634 (2020).

[56] S. S. P. Parkin, M. Hayashi, and L. Thomas, Magnetic domain-wall racetrack memory, Science 320, 190 (2008).

[57] T. Sakudo and H. Unoki, Dielectric Properties of $\mathrm{SrTiO}_{3}$ at Low Temperatures, Phys. Rev. Lett. 26, 851 (1971). 